\title{
Ambulatory blood pressure monitoring: Is 24 hours necessary?
}

\author{
Michael Vornovitsky, Benjamin R. McClintic, G. Ronald Beck, John D. Bisognano \\ Internal Medicine and Cardiology Division, University of Rochester Medical Center, \\ Rochester, New York, United States
}

\begin{abstract}
Background: The variability of blood pressure $(B P)$ makes any single measurement a poor indicator of a patient's true BP. Multiple studies have confirmed the superiority of ambulatory $B P$ measurements over clinic BP measurements in predicting cardiovascular risk; however, this method presents the problem of patient acceptance as it causes frequent arm discomfort and sleep disturbance. We hypothesized that 6 h of daytime BP measurements would result in slightly higher $B P$ readings, yet reveal similar clinical decision making when compared to 24 h of BP measurements.

Methods: The source for writing this article was a retrospective analysis of 30 patients who underwent ambulatory BP monitoring. Data obtained included: age, sex, ethnicity, baseline medical problems, medications, laboratory values, reason given for ordering 24-h ambulatory BP measurements, ambulatory BP measurements, and a subsequent decision to change medication.

Results: The average BP of the 24-h measurements was 127/75 $\mathrm{mm} \mathrm{Hg}$ and the average BP of the 6-h daytime measurements was 131/79 $\mathrm{mm} \mathrm{Hg}$ (SD 15, $p=0.009$ ). Twenty-six out of 30 patients were at goal or pre-hypertensive. Two out of 30 patients had stage 1 hypertension and 2 out of 30 patients had stage 2 hypertension. Thirteen out of 30 patients had nocturnal dipping. Twelve out of 30 patients had a change in medication, but those changes were not associated with the presence or absence of nocturnal dipping $(p=0.5)$ or other factors beyond mean BP. Conclusions: Although there was a statistically significant, $4 \mathrm{~mm} \mathrm{Hg}$ systolic difference between 24- $h$ and 6-h average BP readings, there was no evidence that this difference led to changes in clinical management. The presence or absence of nocturnal dipping was not associated with a change in medication. We conclude that 6-h daytime ambulatory BP measurements provide sufficient information to guide clinical decision making without the problems of patient acceptance, arm discomfort, and sleep disturbance associated with 24-h BP measurements. (Cardiol J 2013; 20, 6: 662-664)
\end{abstract}

Key words: blood pressure monitor, ambulatory

\section{Introduction}

The inherent variability of blood pressure (BP) makes any single measurement a relatively poor indicator of a patient's "true" BP. Furthermore,
BP measurements taken in a physician's office are susceptible to the "white coat" effect and may lead to unnecessary administration of medications. To avoid and/or evaluate these problems, 24-h ambulatory BP monitoring (ABPM) is often used.

Address for correspondence: Dr John Bisognano, University of Rochester Medical Center, 601 Elmwood Avenue, 14642 Rochester, United States, tel: 585-275-6168, e-mail: john_bisognano@urmc.rochester.edu 
Multiple studies have confirmed the superiority of $\mathrm{ABPM}$ over clinic BP measurements in predicting cardiovascular risk [1]. And while the mean 24-h BP has most often been used in studies as a risk predictor as well as in clinical practice, ABPM also allows evaluation of $\mathrm{BP}$ variability and diurnal changes. It is well established that normal subjects have a diurnal rhythm of BP, and they exhibit nocturnal "dipping" of 10-20\% in both their systolic and diastolic BPs while sleeping [2]. Some evidence suggests that nighttime BPs are more predictive of cardiovascular risk than daytime BPs [3, 4], while other data indicate that a diminished nocturnal decline in $\mathrm{BP}$ confers risk above and beyond that of the mean BP [5]. However, conflicting evidence holds that there is no difference in the predictive value of daytime vs. nighttime BPs $[6,7]$.

Despite the well-established role of ABPM in both hypertension diagnosis and measurement of treatment effects, there remain issues of patient acceptance. As compared with self monitoring at home or even clinic measurements, ABPM causes greater arm discomfort and sleep disturbance [8]. Even in a population that expressed high levels of acceptance of the testing, close to one quarter of subjects experienced discomfort from it [9]. Some have tried to determine which specific devices cause less discomfort [10], but to our knowledge there have been no studies examining whether $6 \mathrm{~h}$ of daytime BP measurements provide sufficient data to substitute for a full 24 -h test in everyday clinical practice.

\section{Methods}

This was a retrospective analysis of 30 patients who underwent ABPM in a hypertension clinic during a 12 -month period. Participants had to meet the following inclusion criteria: be at least 18 years of age, complete 24 -h data from the BP recordings, and have documented evidence of a follow-up evaluation with subsequent clinical decision based on the data. For patients with more than one set of 24-h recordings, only their initial test was included in the analysis. Exclusion criteria included: patients whose charts had incomplete data from their 24-h monitoring or patients without documented evidence of a follow-up evaluation after their testing. No exclusions were made based on comorbid illnesses, baseline medications, gender, race, ethnicity, or type of monitor used.

Data obtained included: age, sex, ethnicity, baseline medical problems, medications, laboratory values, reason given for ordering 24-h ambulatory
BP measurements, ambulatory BP measurements, and a subsequent decision to change medication.

Each patient was sent home with an ambulatory BP monitor that recorded BP every hour for a $24 \mathrm{~h}$ period. The mean $24 \mathrm{~h}$ measurement was then compared to the same patient's mean 6 -h daytime $\mathrm{BP}$ (systolic and diastolic) for the same $24 \mathrm{~h}$ period. Each patient's chart was examined for evidence of a change in medication based on 24-h ambulatory $\mathrm{BP}$ readings, and the provider's notations were reviewed with regard to a specific reason for changing medical therapy (e.g., presence of nocturnal dipping, mean systolic or diastolic BPs).

A two-tailed, paired t-test was used to determine statistical significance in mean systolic and mean diastolic BP between 6 -h and 24-h ambulatory monitoring. Fisher's exact test was used to decide whether there was any correlation between nocturnal dipping and change in therapy.

\section{Results}

Thirty patients were included in the statistical analysis. The mean age was 63 . Sixteen (53\%) patients of the participants were female. Twenty-six people were at goal or classified as pre-hypertensive using JNC VII criteria. Two had stage I hypertension and 2 were stage II. The mean 6 h daytime systolic BP was $131 \mathrm{~mm} \mathrm{Hg}$ and diastolic BP was $79 \mathrm{~mm} \mathrm{Hg}$. The mean $24 \mathrm{~h}$ systolic $\mathrm{BP}$ was $127 \mathrm{~mm} \mathrm{Hg}$ and diastolic BP $75 \mathrm{~mm} \mathrm{Hg}$. The difference between $6 \mathrm{~h}$ and $24 \mathrm{~h}$ mean BP measurements was found to be significant $(\mathrm{SD}=15$, $\mathrm{p}=0.009$ ). Over the $24 \mathrm{~h} \mathrm{BP}$ measurement 10 people were noted to have a nighttime dip in BP in the normal BP group, 3 people with stage I hypertension, and none with stage II hypertension. Twelve of the 30 patients received changes in medication regimen, but those changes were not associated with the presence or absence of nocturnal dipping $(p=0.5)$ or other factors beyond mean ambulatory BP measurements (Fig. 1).

\section{Discussion}

The need for ABPM arises from the fact that no single "in office" BP reading accurately reflects hypertension due to a multitude of factors including "white coat" hypertension, episodic hypertension, resistant hypertension, and patient non-adherence to treatment. Misdiagnosis with hypertension leads to unnecessary treatment and possible adverse effects. Though it is more costly to perform ambulatory monitoring, Krakoff [11] concluded that the long term cost of treatment is 


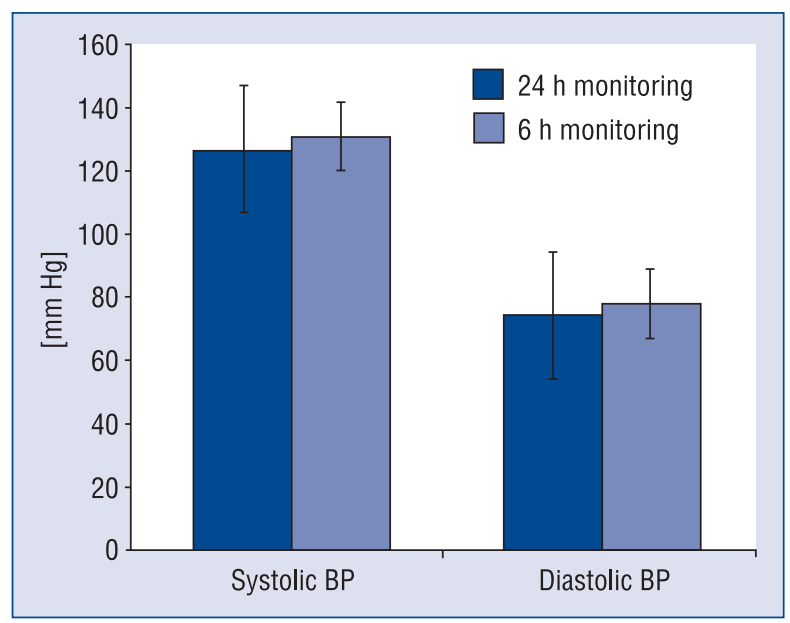

Figure 1. $6 \mathrm{~h}$ and $24 \mathrm{~h}$ mean systolic and diastolic blood pressures (BP).

decreased $3-14 \%$ and treatment days are decreased $10-23 \%$ when ambulatory monitoring is performed. With the establishment of 24 -h recording of BP to diagnose hypertension, several patient specific limitations have arisen. For example, patients may not be compliant with the once hourly recordings for a period of $24 \mathrm{~h}$ due to arm discomfort. It may be especially cumbersome at night due to sleep disturbance. Noncompliance can therefore lead to underdiagnosis or overdiagnosis of hypertension.

\section{Limitations of the study}

The limitations to this study were its small sample size and the use of the same population as both experiment and control. However, the conclusion remains valid due to the need to conserve resources in the face of rising health care costs, increasing patient population, and need for patient satisfaction. As 6-h monitoring is more efficient and potentially less costly, it can be easily incorporated into every day practice.

\section{Conclusions}

This study set out to show that 6-h ambulatory BP monitoring is sufficient to drive clinical judgment on treatment of hypertension. Our results showed that although there was a statistically significant $4 \mathrm{mg} \mathrm{Hg}$ systolic difference between 24- and 6-h recordings, there was no evidence this increase was significant enough to alter treatment. Furthermore, the presence or absence of nocturnal dipping was not associated with changes in medication regimen. Therefore, we concluded that 6 -h daytime ambulatory BP measurements provide sufficient information to guide clinical judgment without the problems of patient acceptance.

\section{Conflict of interest: none declared}

\section{References}

1. Pickering TG, Shimbo D, Haas D. Ambulatory blood-pressure monitoring. N Engl J Med, 2006; 354: 2368-2374.

2. Pickering TG, Harshfield GA, Kleinert HD, Blank S, Laragh JH. Blood pressure during normal daily activities, sleep, and exercise: Comparison of values in normal and hypertensive subjects. JAMA, 1982; 247: 992-996.

3. Dolan E, Stanton A, Thijs L et al. Superiority of ambulatory over clinic blood pressure measurements in predicting mortality: The Dublin outcome study. Hypertension, 2005; 46: 156-161.

4. Staessen JA, Thijs L, Fagard R et al. Predicting cardiovascular risk using conventional vs ambulatory blood pressure in older patients with systolic hypertension. JAMA, 1999; 282: 539-546.

5. Ohkubo T, Hozawa A, Yamaguchi J et al. Prognostic significance of the nocturnal decline in blood pressure in individuals with and without high 24-h blood pressure: The Ohasama study. J Hypertens, 2002; 20: 2183-2189.

6. Bjorklund K, Lind L, Zethelius B, Berglund L, Lithell H. Prognostic significance of 24-h ambulatory blood pressure characteristics for cardiovascular morbidity in a population of elderly men. J Hypertens, 2004; 22: 1691-1697.

7. Khattar RS, Senior R, Lahiri A. Cardiovascular outcome in white-coat versus sustained mild hypertension: A 10-year follow-up study. Circulation, 1998; 98: 1892-1897.

8. Little P, Barnett J, Barnsley L, Marjoram J, Fitzgerald-Barron A, Mant D. Comparison of acceptability of and preferences for different methods of measuring blood pressure in primary care. $\mathrm{Br}$ Med J, 2002; 325: 358-359.

9. Ernst ME, Bergus GR. Favorable patient acceptance of ambulatory blood pressure monitoring in a primary care setting in the United States: A cross-sectional survey. BMC Fam Practice, 2003; 4: 15-20.

10. Westhoff TH, Straub-Hohenbleicher H, Schmidt S et al. Convenience of ambulatory blood pressure monitoring: Comparison of different devices. Clin Methods Path, 2005; 10: 239-242.

11. Krakoff LR. Cost-effectiveness of ambulatory blood pressure: A reanalysis. Hypertension, 2006; 47: 29. 